\title{
CLOSED CONFORMAL VECTOR FIELDS ON PSEUDO-RIEMANNIAN MANIFOLDS
}

\author{
D. A. CATALANO
}

Received 1 March 2006; Revised 24 July 2006; Accepted 8 August 2006

We give here a geometric proof of the existence of certain local coordinates on a pseudoRiemannian manifold admitting a closed conformal vector field.

Copyright (c) 2006 Hindawi Publishing Corporation. All rights reserved.

\section{Introduction}

A vector field $V$ on a pseudo-Riemannian manifold $(M, g)$ is called conformal if

$$
\mathscr{L}_{V} g=2 \lambda g
$$

for a scalar field $\lambda$, where $\mathscr{L}$ denotes the Lie derivative on $M$. It is easy to see that if $V$ is locally a gradient field, then (1.1) is equivalent to

$$
\nabla_{X} V=\lambda X \quad \text { for every vector field } X
$$

Here $\nabla$ denotes the Levi-Civita connection of $g$. We call vector fields satisfying (1.2) closed conformal vector fields. They appear in the work of Fialkow [3] about conformal geodesics, in the works of Yano [7-11] about concircular geometry in Riemannian manifolds, and in the works of Tashiro [6], Kerbrat [4], Kühnel and Rademacher [5], and many other authors.

If $V$ is lightlike on $(M, g)$, then from (1.2), we get

$$
X g(V, V)=2 g\left(\nabla_{X} V, V\right)=2 \lambda g(X, V)=0
$$

for every vector field $X$. Thus $\lambda \equiv 0$ and $V$ is parallel. About lightlike parallel vector fields, we have the following theorem. 
2 Closed conformal vector fields on pseudo-Riemannian manifolds

Theorem 1.1 (Brinkmann [2]). If $(M, g)$ admits a lightlike parallel vector field $V$, then there are local coordinates $u^{1}, u^{2}, \ldots, u^{n}(n:=\operatorname{dim} M>2)$ such that $V=\partial / \partial u^{1}$ and

$$
\left(g_{i j}\right)=\left(\begin{array}{cc|ccc}
0 & 1 & 0 & \ldots & 0 \\
1 & 0 & 0 & \cdots & 0 \\
\hline 0 & 0 & & & \\
\vdots & \vdots & & \left(g_{\alpha \beta}\right) & \\
0 & 0 & & &
\end{array}\right),
$$

where $\alpha, \beta \in\{3, \ldots, n\}$ and $\partial g_{\alpha \beta} / \partial u^{1}=0$.

Brinkmann's proof is purely analytical. We will give, in the next section, geometric tools which will allow us to generalize Brinkmann's theorem.

\section{Geometric constructions}

Let $(M, g)$ be a connected pseudo-Riemannian manifold of dimension $n$ and signature $(k, n-k)$ with $0<k<n$. Given a vector field $W$ on $M$, we denote by $W^{b}$ the one-form defined by $W^{b}(X)=g(W, X)$. Then $W$ is locally a gradient field if and only if $d W^{b}=0$. In the following, a vector field $W$ satisfying $\nabla_{W} W=0$ will be called geodesic.

LEмma 2.1. If $W$ is a geodesic vector field, then $d W^{b}$ is invariant under the flow of $W$.

Proof. Let $\left(\nabla W^{b}\right)(X, Y)=\left(\nabla_{X} W^{b}\right)(Y)=g\left(\nabla_{X} W, Y\right)$. Then, from the fact that $W$ is geodesic, it follows that

$$
\begin{aligned}
\left(\mathscr{L}_{W} \nabla W^{b}\right)(X, Y) & =W g\left(\nabla_{X} W, Y\right)-g\left(\nabla_{[W, X]} W, Y\right)-g\left(\nabla_{X} W,[W, Y]\right) \\
& =g(R(W, X) W, Y)+g\left(\nabla_{X} W, \nabla_{Y} W\right),
\end{aligned}
$$

where $R$ denotes the Riemannian curvature tensor,

$$
R(X, Y) Z=\nabla_{X} \nabla_{Y} Z-\nabla_{Y} \nabla_{X} Z-\nabla_{[X, Y]} Z
$$

Since $g(R(W, X) W, Y)$ is symmetric with respect to $X, Y$, from

$$
d W^{b}(X, Y)=\left(\nabla W^{b}\right)(X, Y)-\left(\nabla W^{b}\right)(Y, X)
$$

we get $\left(\mathscr{L}_{W} d W^{b}\right)(X, Y)=\left(\mathscr{L}_{W} \nabla W^{b}\right)(X, Y)-\left(\mathscr{L}_{W} \nabla W^{b}\right)(Y, X)=0$.

Lemma 2.2. If $W$ is a lightlike geodesic vector field, then $d W^{b}(X, W)=0$. 
Proof. We have the following.

$\left.\begin{array}{l}W \text { lightlike } \Rightarrow\left(\nabla W^{b}\right)(X, W)=g\left(\nabla_{X} W, W\right)=0 \\ W \text { geodesic } \Rightarrow\left(\nabla W^{b}\right)(W, X)=g\left(\nabla_{W} W, X\right)=0\end{array}\right\} \Rightarrow d W^{b}(X, W)=0$.

A nontangent vector field $\widetilde{W}$ on a pseudo-Riemannian hypersurface $\widetilde{M}$ can be extended to a geodesic vector field $W$ in a neighbourhood of $\widetilde{M}$ in the following way. Let $c(s, p)$ be the geodesic starting at $p=c(0, p) \in \widetilde{M}$ with $\dot{c}(0, p)=\widetilde{W}(p)$ and $W(c(s, p)):=$ $\dot{c}(s, p)$. Then, taking into account the fact that $\widetilde{W}$ is transversal (i.e. nontangent) to $\widetilde{M}$, we conclude that $W$ is a geodesic vector field on a neighbourhood of $\widetilde{M}$ extending $\widetilde{W}$. Moreover, if $\widetilde{W}$ is lightlike, then so is $W$. Denoting with $\widetilde{W}^{\top}, \widetilde{W}^{\perp}$ the tangent and normal component of $\widetilde{W}$, for vector fields $X, Y$ on $\widetilde{M}$ tangent to $\widetilde{M}$, we have the following lemma.

Lemma 2.3. $d W^{b}(X, Y)=d\left(\widetilde{W}^{\top}\right)^{b}(X, Y)$.

Proof. The statement follows from $g\left(\nabla_{X} \widetilde{W}^{\perp}, Y\right)-g\left(\nabla_{Y} \widetilde{W}^{\perp}, X\right)=-g\left(\widetilde{W}^{\perp},[X, Y]\right)=0$.

The following remark will be used in the proof of the next proposition.

Remark 2.4. Let $V$ be a vector field and let $\varphi$ be a function on $M$. At a point $p_{0} \in M$, the gradient of the solutions of $V f=\varphi$ span an affine hyperplane $H$ of $T_{p_{0}} M$. Let $v:=V\left(p_{0}\right)$, then $H=\left\{x \in T_{p_{0}} M \mid g(x, v)=\varphi\left(p_{0}\right)\right\}$ and

(a) if $\varphi\left(p_{0}\right) \neq 0$, then $H$ contains lightlike, spacelike, and timelike vectors,

(b) if $\varphi\left(p_{0}\right)=0$, then $H$ contains only lightlike vectors and the zero vector if and only if $n=2$ and $v$ is lightlike.

Proposition 2.5. If $V$ is a closed conformal vector field on $(M, g)$, then in a neighbourhood of a point $p_{0}$ where $V\left(p_{0}\right) \neq 0$, there is a lightlike geodesic gradient field $W$ such that $g(V, W)=1$.

Proof. We divide the proof into two cases.

Case 1. $n>2$ or $n=2$ and $V\left(p_{0}\right)$ is nonlightlike.

Let $u$ be a solution of $V u=0$ with $g\left(p_{0}\right)(\nabla u, \nabla u) \neq 0$ (here $\nabla u$ denotes the gradient of $u$ ). According to Remark 2.4(b), such a solution exists. Let $u$ be an open neighbourhood of $p_{0}$ on which $g(\nabla u, \nabla u) \neq 0$, and let $\widetilde{M}$ be the pseudo-Riemannian hypersurface $u^{-1}\left(u\left(p_{0}\right)\right) \cap u$. Then $\nabla u$ is a normal vector field on $\widetilde{M}$ and, from $V u=0$, we have that $\widetilde{V}:=\left.V\right|_{\widetilde{M}}$ is a tangent vector field on $\widetilde{M}$. Let $\tilde{f}: \widetilde{M} \rightarrow \mathbb{R}$ be a solution of $\tilde{V} \tilde{f}=1$ such that $g\left(p_{0}\right)(\nabla \tilde{f}, \nabla \tilde{f})$ and $g\left(p_{0}\right)(\nabla u, \nabla u)$ have opposite sign (see Remark 2.4(a)). Without loss of generality, we assume that $g(\nabla \tilde{f}, \nabla \tilde{f}) \neq 0$ on $\widetilde{M}$. Setting $\widetilde{W}:=\nabla \tilde{f}+h \nabla u$, where $h^{2}:=-g(\nabla \tilde{f}, \nabla \tilde{f}) / g(\nabla u, \nabla u)>0$, we get

$$
g(\widetilde{W}, \widetilde{W})=g(\nabla \tilde{f}, \nabla \tilde{f})+h^{2} g(\nabla u, \nabla u)=0, \quad g(\tilde{V}, \widetilde{W})=\tilde{V} \tilde{f}=1 .
$$

Let now $W$ be the geodesic vector field extending $\widetilde{W}$ in a neighbourhood of $\widetilde{M}$. Then $W$ is lightlike. From $W g(V, W)=g\left(\nabla_{W} V, W\right)+g\left(V, \nabla_{W} W\right)=0$ and $g(\tilde{V}, \widetilde{W})=1$, we conclude that $g(V, W)=1$. It remains to show that $W$ is locally a gradient. 
4 Closed conformal vector fields on pseudo-Riemannian manifolds

For vector fields $X, Y$ on $\widetilde{M}$ (not necessarily tangent to $\widetilde{M}$ ), we can write

$$
X=X^{\top}+\alpha \widetilde{W}, \quad Y=Y^{\top}+\beta \widetilde{W},
$$

where $\alpha$ and $\beta$ are certain functions on $\widetilde{M}$ and $X^{\top}, Y^{\top}$ are tangent to $\widetilde{M}$. Using Lemma 2.2, we get

$$
0=d W^{b}(X, W)=d W^{b}\left(X^{\top}+\alpha W, W\right)=d W^{b}\left(X^{\top}, W\right)
$$

In the same way, we get $d W^{b}\left(W, Y^{\top}\right)=0$, and therefore $d W^{b}(X, Y)=d W^{b}\left(X^{\top}, Y^{\top}\right)$. Now Lemma 2.3 and $\widetilde{W}^{\top}=\nabla \tilde{f}$ imply that $d W^{b}(X, Y)=0$ on $\widetilde{M}$. Using Lemma 2.1 , we conclude that $d W^{b}=0$.

Case 2. $n=2$ and $V\left(p_{0}\right)$ is lightlike.

According to Remark 2.4(b), we cannot proceed as in Case 1 since the gradient at $p_{0}$ of a solution of $V u=0$ is a lightlike vector. Remarking that along an integral curve $\alpha$ of $V$ through $p_{0} V$ is lightlike, we set $\widetilde{M}:=\operatorname{Im} \alpha$. Let now $\widetilde{W}$ be a lightlike vector field along $\alpha$ such that $V$ and $\widetilde{W}$ are linearly independent. Then, since $g$ is nondegenerate, $g(V, V) g(\widetilde{W}, \widetilde{W})-g(V, \widetilde{W})^{2}=-g(V, \widetilde{W})^{2} \neq 0$. Therefore we can assume that $g(V, \widetilde{W})=$ 1. Since $\widetilde{W}$ is not tangent to $\alpha$, we can extend it to a geodesic vector field $W$ on a neighbourhood $U$ of $p_{0}$. Then $W g(W, W)=0$ which, together with $\widetilde{W}$ lightlike, implies $W$ lightlike, and $W g(V, W)=g\left(\nabla_{W} V, W\right)=0$ which, together with $g(V, \widetilde{W})=1$, implies $g(V, W)=1$. Since every vector field on $U$ can be written as a linear combination of $V$ and $W$, we have $g\left(\nabla_{X} W, Y\right)-g\left(\nabla_{Y} W, X\right)=0$ for every vector field $X, Y$ on $U$ if and only if $g\left(\nabla_{V} W, W\right)-g\left(\nabla_{W} W, V\right)=0$.

Thus $W$ being lightlike and geodesic implies that $W$ is a gradient vector field.

It remains to show that $V$ is lightlike along an integral curve $\alpha$ through $p_{0}:=\alpha(0)$. This follows from $(d / d t) g(V, V)=2 g\left(\nabla_{V} V, V\right)=2 \lambda g(V, V)$, since its general solution is $g(\alpha(t))(V, V)=g\left(p_{0}\right)(V, V) e^{2 \int_{0}^{t} \lambda(u) d u}$.

For example, let $M=\mathbb{R}_{k}^{n}$ be the pseudo-Euclidian space of dimension $n$ and signature $(k, n-k)$ with $0<k<n$, that is, $\langle x, x\rangle=-\left(x_{1}^{2}+\cdots+x_{k}^{2}\right)+\left(x_{k+1}^{2}+\cdots+x_{n}^{2}\right)$. The position vector field $V(x)=\left.\sum_{i=1}^{n} x_{i}\left(\partial / \partial x_{i}\right)\right|_{x}$ satisfies $\nabla_{X} V=X$, and therefore it is a closed conformal vector field. We will construct, following the proof of Proposition 2.5, a lightlike geodesic gradient field $W$ with $\langle V, W\rangle=1$ in a neighbourhood of a point $x_{0} \neq 0(V(x)=0$ if and only if $x=0)$. We take for simplicity $x_{0}=(1,0, \ldots, 0)$, then $u\left(x_{1}, \ldots, x_{n}\right):=x_{n} / x_{1}$ is a solution of $V u=0$ with $\left.\langle\nabla u, \nabla u\rangle\right|_{x_{0}}=1$. The hypersuface $\widetilde{M}:=u^{-1}\left(u\left(x_{0}\right)\right)=u^{-1}(0)$ is the hyperplane $x_{n}=0$. Let $\tilde{V}:=\left.V\right|_{\widetilde{M}}$, then $\tilde{f}\left(x_{1}, \ldots, x_{n-1}\right):=\ln x_{1}$ is a solution of $\tilde{V} \tilde{f}=1$ with $\left.\langle\nabla \tilde{f}, \nabla \tilde{f}\rangle\right|_{x_{0}}=-1$. Defining for every $x \in \widetilde{M}$ that

$$
\widetilde{W}(x):=\nabla \tilde{f}(x)+\nabla u(x)=\left.\frac{1}{x_{1}}\left(-\frac{\partial}{\partial x_{1}}+\frac{\partial}{\partial x_{n}}\right)\right|_{x},
$$

it is easy to see that

$$
W(x):=\left.\frac{1}{x_{1}+x_{n}}\left(-\frac{\partial}{\partial x_{1}}+\frac{\partial}{\partial x_{n}}\right)\right|_{x}
$$


is a geodesic vector field on $M$ extending $\widetilde{W}$. Moreover $W$ is lightlike, $\langle V, W\rangle=1$, and $W=\nabla \ln \left|x_{1}+x_{n}\right|$. It is clear that $W$ is not unique and not everywhere defined. More generally, for an arbitrary point $x_{0} \neq 0$, we have, for instance, that

$$
W=\nabla \ln |\langle a, x\rangle|, \quad \text { where } a \text { is a lightlike vector in } \mathbb{R}_{k}^{n} \text { with }\left\langle a, x_{0}\right\rangle \neq 0,
$$

is a lightlike geodesic gradient field satisfying $\langle V, W\rangle=1$.

Finally we remark that a nontrivial conformal vector field (a vector field $V$ is nontrivial if there is a point $p \in M$ with $V(p) \neq 0$ ) has isolated zeros (see [4]). This is in general not true if the conformal vector field is not closed (see, e.g., an example in [1]).

\section{Local coordinates}

Let $V$ and $W$ be vector fields as in Proposition 2.5 and let $E_{1}=V-g(V, V) W, E_{2}=W$. It is easy to see that

(i) $E_{1}, E_{2}$ are linearly independent;

(ii) the distribution $\mathscr{D}$ spanned by $E_{1}, E_{2}$ is integrable and the metric $g$ is nondegenerate on D;

(iii) the distribution $\mathscr{D}^{\perp}$ spanned by the vector fields orthogonal to $E_{1}, E_{2}$ is integrable and $g$ is nondegenerate on $\mathscr{D}^{\perp}$;

(iv) $\left[E_{1}, E_{2}\right]=0$.

We can now state the following theorem.

THeOREM 3.1. If $(M, g)$ admits a closed conformal vector field $V$, then in a neighbourhood of a point $p_{0}$ where $V\left(p_{0}\right) \neq 0$, there are local coordinates $u^{1}, u^{2}, \ldots, u^{n}$ such that $V=\partial / \partial u^{1}+$ $a\left(\partial / \partial u^{2}\right)$, for some function $a=a\left(u^{2}\right)$, and

$$
\left(g_{i j}\right)=\left(\begin{array}{cc|ccc}
-a & 1 & 0 & \ldots & 0 \\
1 & 0 & 0 & \ldots & 0 \\
\hline 0 & 0 & & & \\
\vdots & \vdots & & \left(g_{\alpha \beta}\right) & \\
0 & 0 & & &
\end{array}\right),
$$

where $\alpha, \beta \in\{3, \ldots, n\}$, $\operatorname{det}\left(g_{\alpha \beta}\right) \neq 0$, and $\partial g_{\alpha \beta} / \partial u^{1}+a\left(\partial g_{\alpha \beta} / \partial u^{2}\right)=a^{\prime} g_{\alpha \beta}\left(a^{\prime}:=d a / d u^{2}\right)$.

Proof. From Frobenius theorem, we know that there are local coordinates $u^{1}, u^{2}, \ldots, u^{n}$ such that

$$
\frac{\partial}{\partial u^{1}}=E_{1}, \quad \frac{\partial}{\partial u^{2}}=E_{2}, \quad g_{1 \alpha}=g_{2 \alpha}=0, \quad \alpha=3, \ldots, n
$$


6 Closed conformal vector fields on pseudo-Riemannian manifolds

Hence $g_{11}=g\left(E_{1}, E_{1}\right)=g(V, V)-2 g(V, V) g(V, W)=-g(V, V), g_{12}=g(V, W)=1, g_{22}=$ $g(W, W)=0$ and, setting $E_{i}=\partial / \partial u^{i}, i=1, \ldots, n$, we have that

$$
\begin{aligned}
\frac{\partial g_{\alpha \beta}}{\partial u^{1}}+a \frac{\partial g_{\alpha \beta}}{\partial u^{2}}= & g\left(\nabla_{E_{1}} E_{\alpha}+g(V, V) \nabla_{E_{2}} E_{\alpha}, E_{\beta}\right) \\
& +g\left(E_{\alpha}, \nabla_{E_{1}} E_{\beta}+g(V, V) \nabla_{E_{2}} E_{\beta}\right) \\
= & g\left(\nabla_{E_{\alpha}} E_{1}+g(V, V) \nabla_{E_{\alpha}} E_{2}, E_{\beta}\right) \\
& +g\left(E_{\alpha}, \nabla_{E_{\beta}} E_{1}+g(V, V) \nabla_{E_{\beta}} E_{2}\right) \\
= & g\left(\nabla_{E_{\alpha}}\left(E_{1}+g(V, V) E_{2}\right), E_{\beta}\right) \\
& +g\left(E_{\alpha}, \nabla_{E_{\beta}}\left(E_{1}+g(V, V) E_{2}\right)\right) \\
= & g\left(\nabla_{E_{\alpha}} V, E_{\beta}\right)+g\left(E_{\alpha}, \nabla_{E_{\beta}} V,\right)=2 \lambda g_{\alpha \beta},
\end{aligned}
$$

where $a=g(V, V)$. From $X g(V, V)=2 \lambda g(X, V)$ and $g\left(E_{1}, V\right)=g\left(E_{3}, V\right)=\cdots=g\left(E_{n}, V\right)=$ 0 , we conclude that $a=a\left(u^{2}\right)$. Furthermore

$$
a^{\prime}=W g(V, V)=2 \lambda
$$

and $a=0$ if and only if $V$ is lightlike (cf. with Brinkmann's theorem).

On the other hand, we have the following proposition.

Proposition 3.2. If on a neighbourhood $\mathcal{U}$ of a point $p_{0} \in M$, there are local coordinates as in Theorem 3.1, then $V=\partial / \partial u^{1}+a\left(\partial / \partial u^{2}\right)$ is a closed conformal vector field on $U$.

Proof. The statement follows from

$$
\begin{aligned}
g\left(\nabla_{E_{i}} V, E_{j}\right) & =g\left(\nabla_{E_{i}} E_{1}, E_{j}\right)+a^{\prime} \delta_{2 i} \delta_{1 j}+a g\left(\nabla_{E_{i}} E_{2}, E_{j}\right) \\
& =\frac{1}{2}\left(\frac{\partial g_{1 j}}{\partial u^{i}}+\frac{\partial g_{i j}}{\partial u^{1}}-\frac{\partial g_{1 i}}{\partial u^{j}}+a \frac{\partial g_{i j}}{\partial u^{2}}\right)+a^{\prime} \delta_{2 i} \delta_{1 j} \\
& =\frac{1}{2}\left(\frac{\partial g_{i j}}{\partial u^{1}}+a \frac{\partial g_{i j}}{\partial u^{2}}\right)+\frac{1}{2} a^{\prime}\left(\delta_{1 i} \delta_{2 j}+\delta_{2 i} \delta_{1 j}\right),
\end{aligned}
$$

where $\delta$ is the Kronecker delta. Namely, for every pair $(i, j)$, we get $g\left(\nabla_{E_{i}} V, E_{j}\right)=$ $(1 / 2) a^{\prime} g_{i j}$. Moreover, $V$ is lightlike if and only if $a=0$.

Remark 3.3. If in Proposition 3.2 we assume that $a \neq 0$, then according to Fialkow results, see [3, formulas (12.9) and (12.10)], we must be able to prove that $(u, g)$ is locally isometric to a warped product with a one-dimensional base manifold. This can be seen in 
the following way: take local coordinates $\bar{u}^{1}, \ldots, \bar{u}^{n}$ in $U$ such that

$$
\frac{\partial}{\partial \bar{u}^{1}}=\frac{1}{\sqrt{|a|}}\left(\frac{\partial}{\partial u^{1}}+a \frac{\partial}{\partial u^{2}}\right), \quad \frac{\partial}{\partial \bar{u}^{2}}=\frac{\partial}{\partial u^{1}}, \quad \frac{\partial}{\partial \bar{u}^{\alpha}}=\frac{\partial}{\partial u^{\alpha}}, \quad \alpha=3, \ldots, n .
$$

This is reached by the coordinate transformation

$$
\bar{u}^{1}=\int \frac{\sqrt{|a|}}{a} d u^{2}, \quad \bar{u}^{2}=u^{1}-\int \frac{1}{a} d u^{2}, \quad \bar{u}^{\alpha}=u^{\alpha}, \quad \alpha=3, \ldots, n .
$$

Then it is easy to see that $a=a\left(\bar{u}^{1}\right)$ and that

$$
\left(\bar{g}_{i j}\right):=\left(g\left(\frac{\partial}{\partial \bar{u}^{i}}, \frac{\partial}{\partial \bar{u}^{j}}\right)\right)=\left(\begin{array}{c|cccc} 
\pm 1 & 0 & 0 & \cdots & 0 \\
\hline 0 & -a & 0 & \cdots & 0 \\
0 & 0 & & & \\
\vdots & \vdots & & \left(g_{\alpha \beta}\right) & \\
0 & 0 & & &
\end{array}\right) .
$$

Furthermore, from $\partial g_{\alpha \beta} / \partial u^{1}+a\left(\partial g_{\alpha \beta} / \partial u^{2}\right)=a^{\prime} g_{\alpha \beta}$, we get

$$
\frac{\partial g_{\alpha \beta}}{\partial \bar{u}^{1}}=\frac{1}{\sqrt{|a|}}\left(\frac{\partial g_{\alpha \beta}}{\partial u^{1}}+a \frac{\partial g_{\alpha \beta}}{\partial u^{2}}\right)=\frac{1}{\sqrt{|a|}} \frac{d a}{d u^{2}} g_{\alpha \beta}=\frac{1}{a} \frac{d a}{d \bar{u}^{1}} g_{\alpha \beta},
$$

and therefore $g_{\alpha \beta}=a \bar{g}_{\alpha \beta}$, where $\partial \bar{g}_{\alpha \beta} / \partial \bar{u}^{1}=0$. Thus $(\vartheta, g)$ is locally isometric to a warped product with a one-dimensional base manifold and warped factor $a$. In these local coordinates, the metric of the fiber manifold is given by

$$
\left(\begin{array}{c|ccc}
-1 & 0 & \cdots & 0 \\
\hline 0 & & & \\
\vdots & & \left(\bar{g}_{\alpha \beta}\right) & \\
0 & & &
\end{array}\right)
$$

which means, in other words, that $\bar{u}^{2}, \ldots, \bar{u}^{n}$ are Fermi coordinates on the fiber manifold.

\section{Acknowledgment}

The author wishes to thank Professor K. Voss of the Swiss Federal Institute of Technology in Zurich for helpful suggestions on the subject.

\section{References}

[1] D. Alekseevski, Self-similar Lorentzian manifolds, Annals of Global Analysis and Geometry 3 (1985), no. 1, 59-84.

[2] H. W. Brinkmann, Einstein spaces which are mapped conformally on each other, Mathematische Annalen 94 (1925), no. 1, 119-145.

[3] A. Fialkow, Conformal geodesics, Transactions of the American Mathematical Society 45 (1939), no. 3, 443-473. 
[4] Y. Kerbrat, Transformations conformes des variétés pseudo-riemanniennes, Journal of Differential Geometry 11 (1976), no. 4, 547-571.

[5] W. Kühnel and H.-B. Rademacher, Essential conformal fields in pseudo-Riemannian geometry, Journal de Mathématiques Pures et Appliquées. Neuvième Série 74 (1995), no. 5, 453-481.

[6] Y. Tashiro, Complete Riemannian manifolds and some vector fields, Transactions of the American Mathematical Society 117 (1965), 251-275.

[7] K. Yano, Concircular geometry III. Theory of curves, Proceedings of the Imperial Academy of Tokyo 16 (1940), 442-448.

[8] Concircular geometry IV. Theory of subspaces, Proceedings of the Imperial Academy of Tokyo 16 (1940), 505-511.

[9] _ Concircular geometry. I. Concircular transformations, Proceedings of the Imperial Academy of Tokyo 16 (1940), 195-200.

[10] Concircular geometry. II. Integrability conditions of $\rho_{\mu \nu}=\varphi g_{\mu \nu}$, Proceedings of the Imperial Academy of Tokyo 16 (1940), 354-360.

[11] Concircular geometry. V. Einstein spaces, Proceedings of the Imperial Academy of Tokyo 18 (1942), 446-451.

D. A. Catalano: Departamento de Matemática, Universidade de Aveiro, Campus de Santiago, 3810-193 Aveiro, Portugal

E-mail address: domenico@mat.ua.pt 


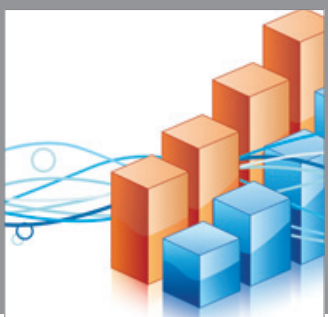

Advances in

Operations Research

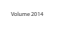

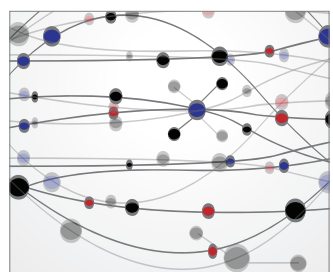

\section{The Scientific} World Journal
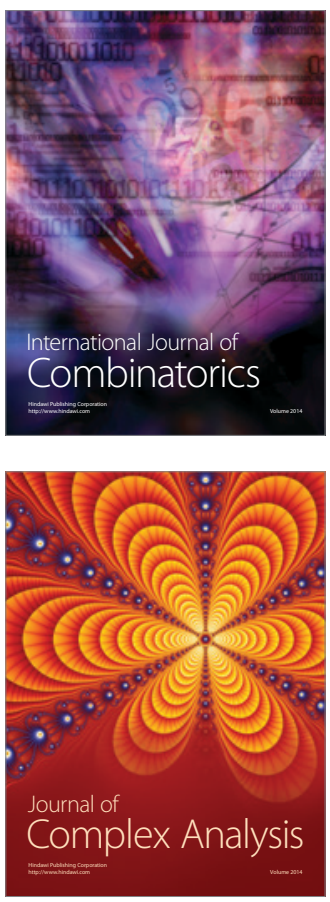

International Journal of

Mathematics and

Mathematical

Sciences
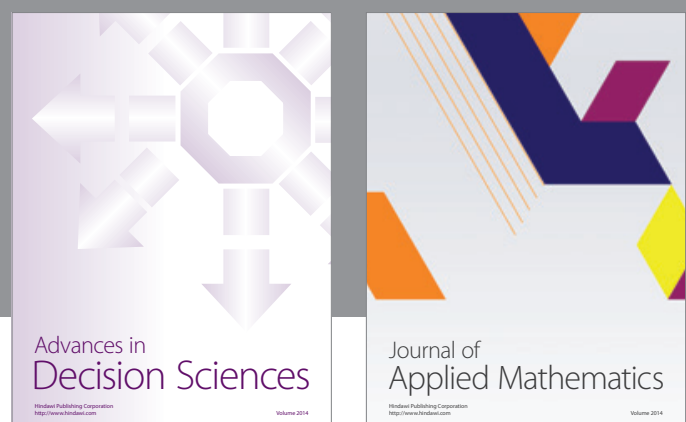

Journal of

Applied Mathematics
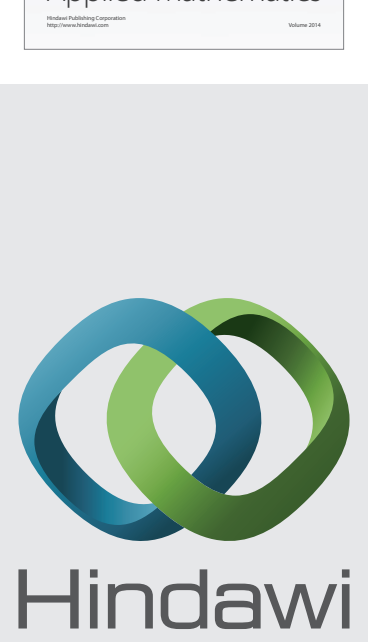

Submit your manuscripts at http://www.hindawi.com
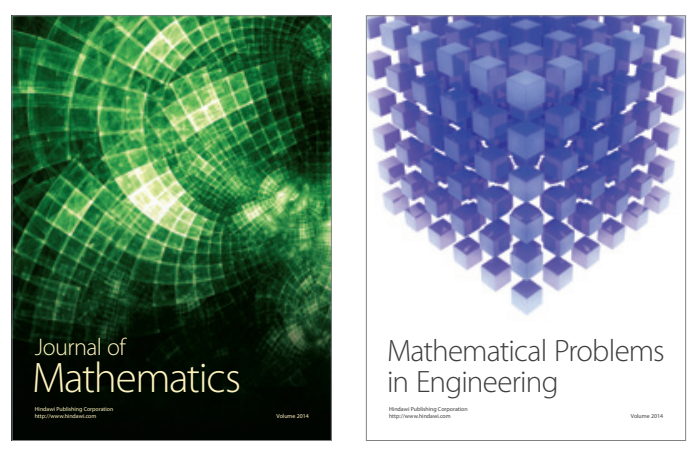

Mathematical Problems in Engineering
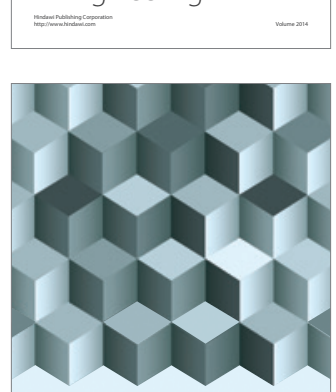

Journal of

Function Spaces
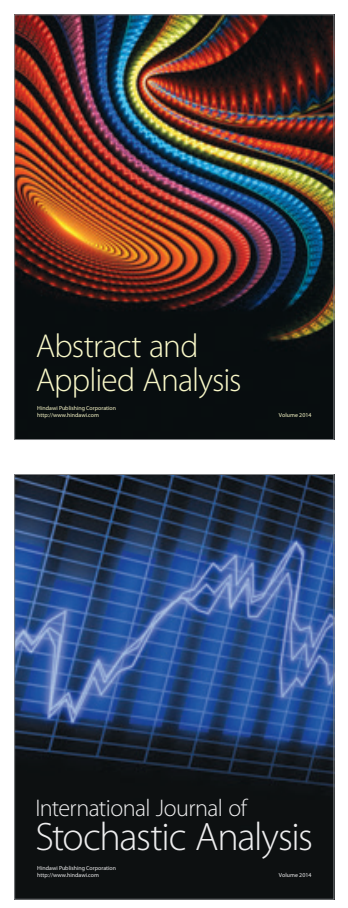

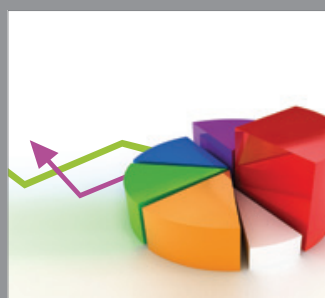

ournal of

Probability and Statistics

Promensencen
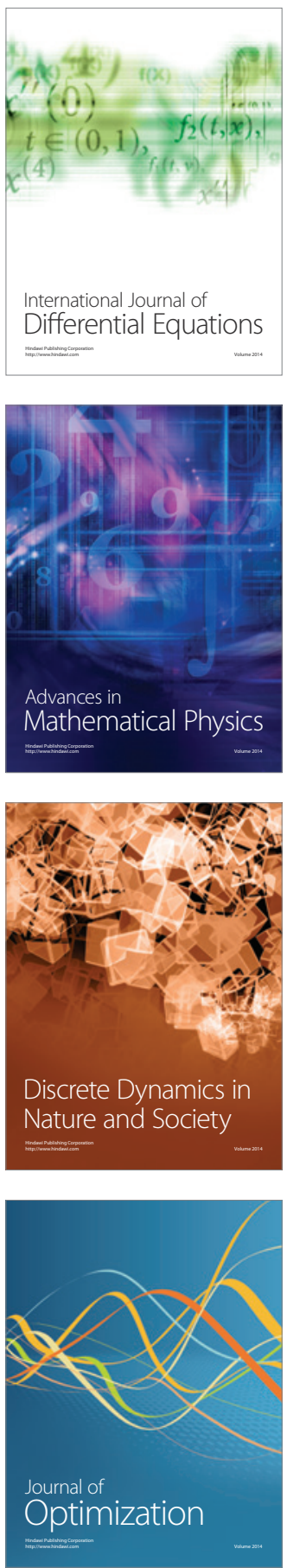